\title{
Exploration, Characterization and Conservation of Cotton (Gossypium spp.) Encompassing Colour Cotton from Tripura, India
}

\author{
M. Saravanan ${ }^{1}$, R. C. Misra ${ }^{2}$, S. S. Mahajan ${ }^{1}$, P. Mohan ${ }^{1}$ and V. N. Waghmare ${ }^{1 *}$ \\ ${ }^{1}$ ICAR-Central Institute for Cotton Research, Nagpur, Maharashtra, India \\ ${ }^{2}$ ICAR-National Bureau of Plant Genetic Resources, Regional Station, Cuttack-753006, \\ Odisha, India \\ *Corresponding author
}

\section{A B S T R A C T}

\section{Keywords}

Characterization, Colour cotton, Desi cotton, Exploration, Tripura

\section{Article Info}

Accepted:

22 January 2021

Available Online:

10 February 2021
Exploration survey was conducted in Tripura state to collect, characterize and conserve the valuable traditional cultivars of desi cotton. Fourteen germplasm accessions comprising ten Gossypium arboreum L. and four Gossypium barbadense L. were collected from Gomati, Khowai, West Tripura and Dhalai districts of Tripura. The collected accessions of desi cotton (G. arboreum) represent derivatives of race 'cernuum' and 'burmanicum'. The morphological variants of desi cotton were compact, having short sympodia, medium size boll with short staple fibre. The range for fibre quality traits such as fibre length (17.2 $19.1 \mathrm{~mm})$, fibre strength $(15.0$ - $16.7 \mathrm{~g} / \mathrm{tex})$, micronaire $(>7)$, Elongation Index (4.1 - 4.6) and Short Fibre Index (45.1 - 61.4) was recorded for desi cotton. Among G. arboreum accessions, two were naturally coloured light brown and brown linted cotton. The range of fibre quality traits with respect to fibre length $(32.6-34.9 \mathrm{~mm})$, micronaire $(5.8-6.4)$ and fibre strength (21.6 - $24.7 \mathrm{~g} / \mathrm{tex})$ was recorded in $G$. barbadense collection. The cotton accessions were conserved in the Field Gene Bank and medium term cold storage at ICAR-Central Institute for Cotton Research, Nagpur for future utilization in cotton improvement.

\section{Introduction}

Cotton is an important fibre, animal feed and oil seed crop. Because of its commercial value, it is popularly referred as "White Gold". India is the sole country in the world to grow all four cultivated species which includes two diploids $(2 \mathrm{n}=2 \mathrm{x}=26) \quad G$. arboreum and $G$. herbaceum and two tetraploids $(2 \mathrm{n}=2 \mathrm{x}=52) G$. hirsutum and $G$. barbadense. India is considered to be the centre of origin for cultivated Asiatic species Gossypium arboreum L., that reported to be domesticated into three geographical races, namely bengalense, cernuum and indicum (Hutchinson et al., 1947). The dispersal of race 'indicum' is considered eastward to north eastern India and Myanmar formed another race 'burmanicum' (Kulkarni et al., 2009). They possess invaluable genetic resources of innumerable genes that confer resistance to biotic and abiotic stresses such as ability to 
withstand drought, heat, salinity and sodicity (Maqbool et al., 2010; Tahir et al., 2011); tolerance to pests and disease, including bollworms (Dhawan et al., 1991); aphids, leaf hoppers (Nibouche et al., 2008); rust, fungi (Wheeler et al., 1999); viral diseases (Mehetre et al., 2004; Akhtar et al., 2010) and many adaptive features.

Tripura is one of the seven sisters that form the North-Eastern Region of India. It is a tribal dominated state in this region. Jhum cultivation (slash-burn-sow method of agriculture) is the major traditional method of cultivation which is primarily adopted since generations. Cotton is always being cultivated as a mixed crop instead of sole crop. The other cultivated crop includes maize, gingely, red gram and different vegetables. Farmers from NEH region have been cultivating desi cotton including coloured cotton since ages. In India, natural desi cotton was popular in some states viz., Karnataka, Andhra Pradesh and West Bengal. Manipur Khaki, Coconadas-1 and Coconadas-2 were important cultivars grown in our country and particularly Coconadas were exported to Japan at premium prices (Kranthi, 2014). Due to low productivity per unit area, poor fibre characteristics and nonuniformity colours, colour cotton cultivation in India was totally replaced by white linted genotypes (Waghmare and Koranne, 1998). Because of the artificial dying in the textile sector, the chemicals are polluting the river and aquatic ecosystem including the ground water resources to some extent. Now there is growing concern about sustainable agriculture and management of aquatic ecosystem. Therefore, coloured cotton is highly suitable for cultivation of sustainable organic agriculture and also useful for export oriented eco-friendly garments. The objective of exploration was to collect, characterize and conserve the germplasm and traditional cultivars of cotton for crop improvement studies.

\section{Materials and Methods}

\section{Exploration and collection survey of cotton from Tripura}

Based on information from literature survey and gap analysis, ICAR-National Bureau of Plant Genetic Resources, New Delhi in collaboration with ICAR-Central Institute for Cotton Research, Nagpur conducted an expedition survey to collect primitive cultivars of cotton from the state of Tripura (Fig. 1). All standard principles and procedures including sampling techniques were followed in our exploration study as per the guidelines laid out by ICAR-NBPGR, New Delhi (Pareek et al., 2000). At the collection site, identification and preliminary characterization was carried as per the Germplasm Index Card formulated by ICARCICR, Nagpur which includes a range of morphological characteristics, response to diseases and pests.

\section{Morphological characterization}

The collected cotton accessions were sown in augmented design at ICAR-CICR main research farm, Panjari, Nagpur for characterization (Fig. 2). Agro-morphological traits including DUS characterization was recorded taking five plants of each accession for 30 characters. After ginning, the samples were sent to Ginning Training Centre (GTC), ICAR-Central Institute for Research on Cotton Technology, Nagpur for fibre quality analysis. The germplasm of collected accessions were conserved in the midterm storage module of ICAR- CICR, Nagpur.

\section{Results and Discussion}

The yield levels have reached a plateau due to the narrow genetic base in cotton. To broaden the genetic base for further improvement, it is necessary to collect, characterize, evaluate, 
conserve, document and utilize the genetic diversity. Based on gap analysis and literature survey, an exploration survey was undertaken in Gomati, Khowai, West Tripura and Dhalai districts of Tripura. Fourteen morphological variants of traditional cotton including two germplasm accessions representing desi coloured cotton (Fig. 3) were collected from diverse collection sites such as jhum cultivated areas, homesteads/ kitchen gardens, bunds of nala, farm/ store etc. Agromorphological characterisation including DUS attributes was carried out for all cotton accessions at the institute (Table 1). The desi cotton variants were compact types, having short sympodia, medium size boll with short staple and coarse fibre. The collected $G$. arboreum accessions represent derivatives of race 'cernuum' and race 'burmanicum' (Fig. 4). These accessions bore long bursted bolls measuring up to $15 \mathrm{~cm}$. The range of fibre quality traits recorded for desi cotton with respect to fibre length that varies from 17.2 to $19.1 \mathrm{~mm}$, fibre strength from 15.0 to 16.7 $\mathrm{g} /$ tex, micronaire up to $>7$, Elongation Index from 4.1 to 4.6 and Short Fibre Index from 45.1 to 61.4. Among collected desi cotton, two accessions viz. RCM/PM/MS-8 and RCM/PM/MS-9 were naturally coloured having light brown to brown linted fibres. A total of 33 samples are collected from West Garo hills of North East Hill region that represents a range of characteristics such as large bracts, long petiole, elongated capsule, early maturity, burst capsule length up to 7 inches and bollworm tolerance.

Table.1 Variability index for morpho-economic characters

\begin{tabular}{|c|c|c|c|c|c|c|c|c|}
\hline $\begin{array}{l}\text { Sl. } \\
\text { No }\end{array}$ & Acc. number & Species & $\begin{array}{l}\text { Habit/ } \\
\text { Leaf }\end{array}$ & Flower & Fruit & Seed & $\begin{array}{l}\text { Lint } \\
\text { colour }\end{array}$ & $\begin{array}{c}\text { Average } \\
\text { boll } \\
\text { weight }\end{array}$ \\
\hline 1. & $\begin{array}{l}\text { RCM/PM/MS- } \\
1\end{array}$ & $\begin{array}{l}\text { Gossypium } \\
\text { arboreum }\end{array}$ & $\begin{array}{l}\text { Annual; } \\
\text { deeply } \\
\text { palmate, } \\
\text { lobed, with } \\
\text { short sinus }\end{array}$ & $\begin{array}{l}\text { Corolla yellow, } 5 \\
\text { petals, } \\
\text { pollen yellow, } \\
\text { petal spot present, } \\
\text { bracteoles } 3 \text {, } \\
\text { broad, triangular, } \\
\text { persistent, calyx } \\
\text { cup shaped }\end{array}$ & $\begin{array}{l}\text { Capsule oval } \\
\text { to oblong, } \\
\text { pointed beak, } \\
\text { 3-4 locular }\end{array}$ & $\begin{array}{l}\text { Pointed } \\
\text { apex, } \\
\text { ovoid to } \\
\text { globose }\end{array}$ & White & $4.5-5.0$ \\
\hline 2. & $\begin{array}{l}\text { RCM/PM/MS- } \\
2\end{array}$ & $\begin{array}{l}\text { Gossypium } \\
\text { arboreum }\end{array}$ & $\begin{array}{l}\text { Annual, } \\
\text { deeply } \\
\text { palmate, } \\
\text { lobed, deep } \\
\text { sinus }\end{array}$ & $\begin{array}{l}\text { Corolla yellow, } 5 \\
\text { petals, } \\
\text { pollen yellow, } \\
\text { petal spot present, } \\
\text { bracteoles-3, } \\
\text { broad, triangular, } \\
\text { persistent, calyx } \\
\text { cup shaped }\end{array}$ & $\begin{array}{l}\text { Capsule oval } \\
\text { to oblong, } \\
\text { pointed beak, } \\
\text { 3-4 locular }\end{array}$ & $\begin{array}{l}\text { Pointed } \\
\text { apex, } \\
\text { ovoid to } \\
\text { globose }\end{array}$ & White & $3.8-5.0$ \\
\hline 3. & $\begin{array}{l}\text { RCM/PM/MS- } \\
3\end{array}$ & $\begin{array}{l}\text { Gossypium } \\
\text { arboreum }\end{array}$ & $\begin{array}{l}\text { Annual, } \\
\text { palmate, deep } \\
\text { sinus }\end{array}$ & $\begin{array}{l}\text { Corolla yellow, } 5 \\
\text { petals, } \\
\text { pollen yellow, } \\
\text { petal spot present, } \\
\text { bracteoles-3, } \\
\text { broad, triangular, } \\
\text { persistent, calyx } \\
\text { cup shaped }\end{array}$ & $\begin{array}{l}\text { Capsule oval } \\
\text { to oblong, } \\
\text { pointed beak, } \\
\text { 3- locular }\end{array}$ & $\begin{array}{l}\text { Pointed } \\
\text { apex, } \\
\text { ovoid to } \\
\text { globose }\end{array}$ & White & $2.5-4.3$ \\
\hline 4. & $\begin{array}{l}\mathrm{RCM} / \mathrm{PM} / \mathrm{MS}- \\
4\end{array}$ & $\begin{array}{l}\text { Gossypium } \\
\text { arboreum }\end{array}$ & $\begin{array}{l}\text { Annual, } \\
\text { palmate, deep } \\
\text { sinus }\end{array}$ & $\begin{array}{l}\text { Corolla } \text { yellow, } 5 \\
\text { petals, } \\
\text { pollen } \quad \text { yellow, }\end{array}$ & $\begin{array}{l}\text { Capsule oval } \\
\text { to oblong, } \\
\text { pointed beak, }\end{array}$ & $\begin{array}{l}\text { Pointed } \\
\text { apex, } \\
\text { ovoid to }\end{array}$ & White & $2.5-5.0$ \\
\hline
\end{tabular}




\begin{tabular}{|c|c|c|c|c|c|c|c|c|}
\hline & & & & $\begin{array}{l}\text { petal spot present, } \\
\text { bracteoles- } 3 \text {, } \\
\text { broad, triangular, } \\
\text { persistent, calyx } \\
\text { cup shaped }\end{array}$ & 3 -locular & globose & & \\
\hline 5. & $\begin{array}{l}\text { RCM/PM/MS- } \\
8\end{array}$ & $\begin{array}{l}\text { Gossypium } \\
\text { arboreum }\end{array}$ & $\begin{array}{l}\text { Annual, } \\
\text { deeply } \\
\text { palmate, } \\
\text { lobed with } \\
\text { short sinus }\end{array}$ & $\begin{array}{l}\text { Corolla yellow, } \\
\text { 5petals, } \\
\text { pollen yellow, } \\
\text { long claw, petal } \\
\text { spot present, } \\
\text { bracteoles-3 broad, } \\
\text { triangular, } \\
\text { persistent, calyx- } \\
\text { cup shaped }\end{array}$ & $\begin{array}{l}\text { Capsule oval } \\
\text { to oblong, } \\
\text { pointed beak, } \\
3 \text {-locular }\end{array}$ & $\begin{array}{l}\text { Pointed } \\
\text { apex, } \\
\text { ovoid to } \\
\text { globose }\end{array}$ & $\begin{array}{l}\text { Light } \\
\text { brown }\end{array}$ & $3.5-4.8$ \\
\hline 6. & $\begin{array}{l}\text { RCM/PM/MS- } \\
9\end{array}$ & $\begin{array}{l}\text { Gossypium } \\
\text { arboreum }\end{array}$ & $\begin{array}{l}\text { Annual, } \\
\text { deeply } \\
\text { palmate, leaf } \\
\text { lobed with } \\
\text { short sinus }\end{array}$ & $\begin{array}{l}\text { Corolla yellow, } 5 \\
\text { petals, } \\
\text { pollen yellow, } \\
\text { long claw, petal } \\
\text { spot present, } \\
\text { bracteoles-3 broad, } \\
\text { triangular, } \\
\text { persistent, calyx- } \\
\text { cup shaped }\end{array}$ & $\begin{array}{l}\text { Capsule oval } \\
\text { to oblong, } \\
\text { pointed beak, } \\
3 \text {-locular }\end{array}$ & $\begin{array}{l}\text { Pointed } \\
\text { apex, } \\
\text { ovoid to } \\
\text { globose }\end{array}$ & Brown & $3.5-5.0$ \\
\hline 7. & $\begin{array}{l}\text { RCM/PM/MS- } \\
11\end{array}$ & $\begin{array}{l}\text { Gossypium } \\
\text { arboreum }\end{array}$ & $\begin{array}{l}\text { Annual, } \\
\text { palmate, } 4-5 \\
\text { lobed, } \\
\text { accessory } \\
\text { lobe absent, } \\
\text { short sinus }\end{array}$ & $\begin{array}{l}\text { Corolla yellow, } 5 \\
\text { petals, } \\
\text { pollen yellow, } \\
\text { long claw, petal } \\
\text { spot present, } \\
\text { bracteoles-3 broad, } \\
\text { triangular, } \\
\text { persistent, calyx- } \\
\text { cup shaped }\end{array}$ & $\begin{array}{l}\text { Capsule oval } \\
\text { to oblong, } \\
\text { pointed beak, } \\
\text { 3-4 locular }\end{array}$ & $\begin{array}{l}\text { Pointed } \\
\text { apex, } \\
\text { ovoid to } \\
\text { globose }\end{array}$ & White & $4.0-5.5$ \\
\hline 8. & $\begin{array}{l}\text { RCM/PM/MS- } \\
12\end{array}$ & $\begin{array}{l}\text { Gossypium } \\
\text { arboreum }\end{array}$ & $\begin{array}{l}\text { Annual, } \\
\text { palmate, } 4-5 \\
\text { lobed, } \\
\text { accessory } \\
\text { lobe absent, } \\
\text { short sinus }\end{array}$ & $\begin{array}{l}\text { Corolla yellow, } 5 \\
\text { petals, } \\
\text { pollen yellow, } \\
\text { long claw, petal } \\
\text { spot present, } \\
\text { bracteoles-3 broad, } \\
\text { triangular, } \\
\text { persistent, calyx- } \\
\text { cup shaped }\end{array}$ & $\begin{array}{l}\text { Capsule oval } \\
\text { to oblong, } \\
\text { pointed beak, } \\
\text { 3-4 locular }\end{array}$ & $\begin{array}{l}\text { Pointed } \\
\text { apex, } \\
\text { ovoid to } \\
\text { globose }\end{array}$ & White & $3.8-5.0$ \\
\hline 9. & $\begin{array}{l}\text { RCM/PM/MS- } \\
13\end{array}$ & $\begin{array}{l}\text { Gossypium } \\
\text { arboreum }\end{array}$ & $\begin{array}{l}\text { Annual, } \\
\text { palmate, 4-5 } \\
\text { lobed, } \\
\text { accessory } \\
\text { lobe absent, } \\
\text { short sinus }\end{array}$ & $\begin{array}{l}\text { Corolla yellow, } 5 \\
\text { petals, } \\
\text { pollen yellow, } \\
\text { long claw, petal } \\
\text { spot present, } \\
\text { bracteoles-3 broad, } \\
\text { triangular, } \\
\text { persistent, calyx- } \\
\text { cup shaped }\end{array}$ & $\begin{array}{l}\text { Capsule oval } \\
\text { to oblong, } \\
\text { pointed beak, } \\
\text { 3-4 locular }\end{array}$ & $\begin{array}{l}\text { Pointed } \\
\text { apex, } \\
\text { ovoid to } \\
\text { globose }\end{array}$ & White & $4.0-5.0$ \\
\hline 10. & $\begin{array}{l}\mathrm{RCM} / \mathrm{PM} / \mathrm{MS}- \\
15\end{array}$ & $\begin{array}{l}\text { Gossypium } \\
\text { arboreum }\end{array}$ & $\begin{array}{l}\text { Annual, } \\
\text { palmate, 4-5 } \\
\text { lobed, } \\
\text { accessory } \\
\text { lobe absent, }\end{array}$ & $\begin{array}{l}\text { Corolla yellow, } 5 \\
\text { petals, } \\
\text { pollen yellow, } \\
\text { short claw, petal } \\
\text { spot present, }\end{array}$ & $\begin{array}{l}\text { Capsule oval } \\
\text { to oblong, } \\
\text { pointed beak, } \\
\text { 3-4 locular }\end{array}$ & $\begin{array}{l}\text { Pointed } \\
\text { apex, } \\
\text { ovoid to } \\
\text { globose }\end{array}$ & White & $3.8-5.5$ \\
\hline
\end{tabular}




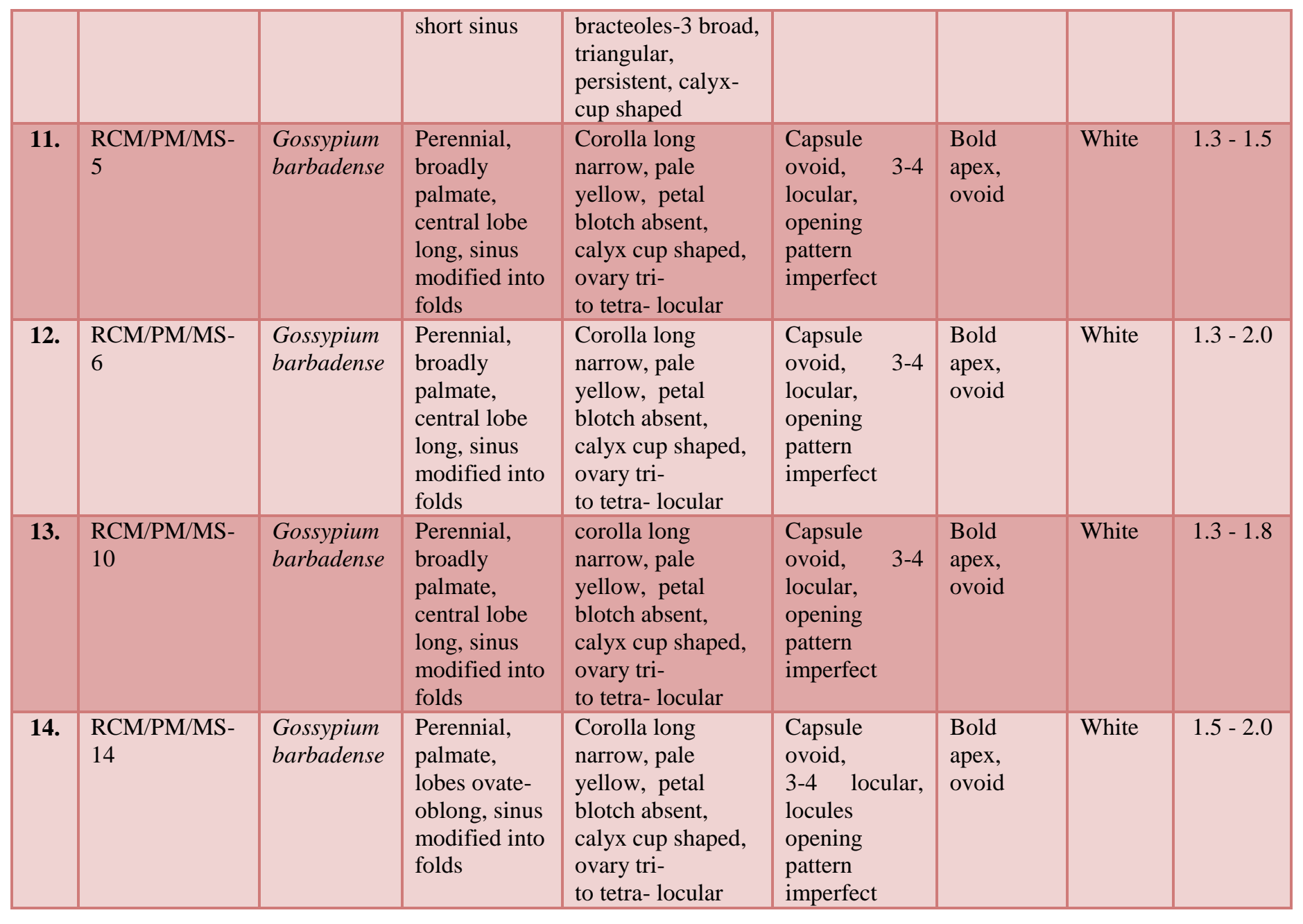

Table.2 Variability index in fibre quality traits

\begin{tabular}{|c|c|c|c|c|c|c|c|}
\hline SI. No. & Collector No. & $\begin{array}{c}\mathbf{2 . 5 \%} \mathbf{~ S L} \\
\text { length } \\
\mathbf{( m m})\end{array}$ & $\begin{array}{c}\text { Uniformi } \\
\text { ty ratio } \\
\mathbf{( \% )}\end{array}$ & $\begin{array}{c}\text { Fineness } \\
\text { micronaire } \\
\mathbf{1 0 - 6} \text { g/in }\end{array}$ & $\begin{array}{c}\text { Bundle strength } \\
\text { tenacity (g/tex) at 3.2 } \\
\text { mm gauge }\end{array}$ & $\begin{array}{c}\text { Elongation } \\
\text { index } \\
\text { \% }\end{array}$ & $\begin{array}{c}\text { Short fibre } \\
\text { index }\end{array}$ \\
\hline $\mathbf{1 .}$ & RCM/PM/MS-1 & 17.3 & 56 & $>7.0$ & 15.9 & 4.3 & 61.4 \\
\hline $\mathbf{2 .}$ & RCM/PM/MS-2 & 17.2 & 56 & $>7.0$ & 15.2 & 4.2 & 60.0 \\
\hline $\mathbf{3 .}$ & RCM/PM/MS-3 & 17.6 & 55 & $>7.0$ & 16.7 & 4.1 & 55.3 \\
\hline $\mathbf{4 .}$ & RCM/PM/MS-4 & 19.1 & 55 & $>7.0$ & 16.3 & 4.2 & 49.5 \\
\hline $\mathbf{5 .}$ & RCM/PM/MS-8 & 17.6 & 56 & $>7.0$ & 15.0 & 4.6 & 51.4 \\
\hline $\mathbf{6 .}$ & RCM/PM/MS-9 & 17.6 & 56 & $>7.0$ & 15.4 & 4.2 & 55 \\
\hline $\mathbf{7 .}$ & RCM/PM/MS-11 & 19.0 & 53 & $>7.0$ & 15.7 & 4.2 & 45.1 \\
\hline $\mathbf{8 .}$ & RCM/PM/MS-12 & 17.2 & 56 & $>7.0$ & 15.1 & 4.3 & 59.4 \\
\hline $\mathbf{9 .}$ & RCM/PM/MS-13 & 17.8 & 57 & $>7.0$ & 16.6 & 4.2 & 56.8 \\
\hline $\mathbf{1 0 .}$ & RCM/PM/MS-15 & 19.0 & 53 & $>7.0$ & 15.7 & 4.1 & 54.2 \\
\hline $\mathbf{1 1 .}$ & RCM/PM/MS-5 & 32.6 & 49 & 6.1 & 23.9 & 5.7 & 4.7 \\
\hline $\mathbf{1 2 .}$ & RCM/PM/MS-6 & 33.6 & 48 & 5.8 & 23.0 & 5.8 & 5.1 \\
\hline $\mathbf{1 3 .}$ & RCM/PM/MS-10 & 34.9 & 51 & 6.0 & 24.7 & 5.5 & 4.4 \\
\hline $\mathbf{1 4 .}$ & RCM/PM/MS-14 & 33.0 & 48 & 6.4 & 21.6 & 5.3 & 5.0 \\
\hline & Range & $\mathbf{1 7 . 2 - 3 4 . 9}$ & $\mathbf{4 8 - 5 7}$ & $\mathbf{5 . 8}->\mathbf{7}$ & $\mathbf{1 5 . 0 - 2 4 . 7}$ & $\mathbf{4 . 1 - 5 . 8}$ & $\mathbf{4 . 4 - 6 1 . 4}$ \\
\hline
\end{tabular}


Fig.1 Collection sites of cotton (Gossypium spp.) germplasm accessions from Tripura, India
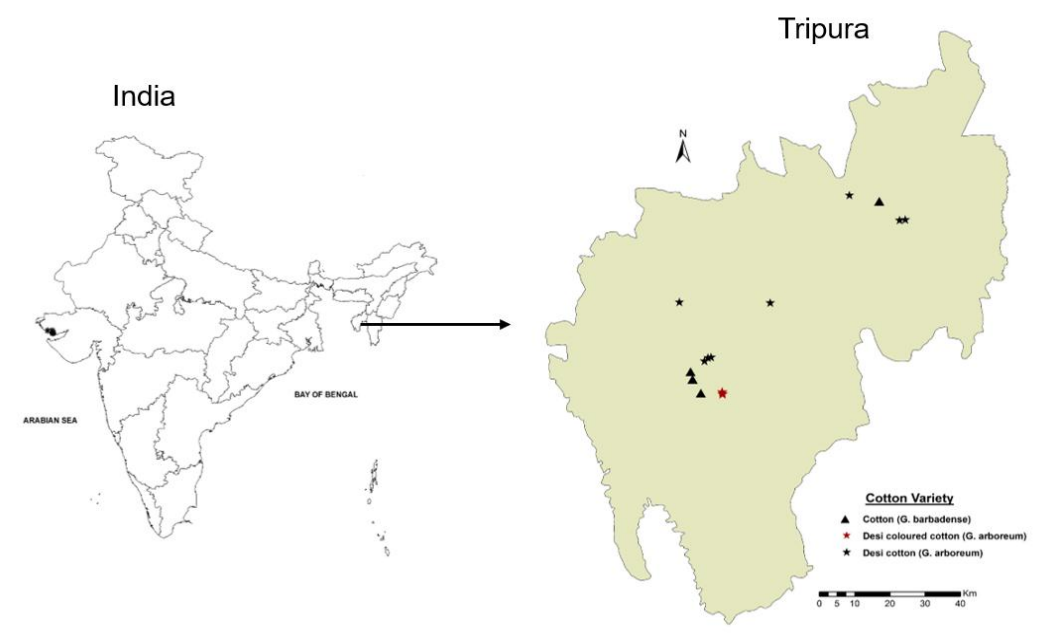

Fig.2 Desi cotton accessions (Gossypium arboreum) in experimental field at Pajri farm, ICARCICR, Nagpur

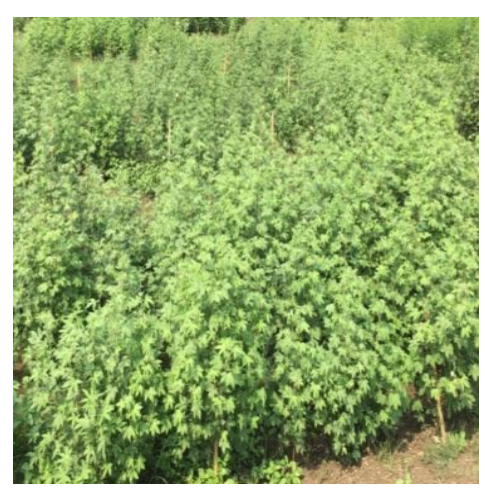

Fig.3 a. Desi colour cotton plant (Gossypium arboreum) at Kalsibari village, Gomati district, Tripura

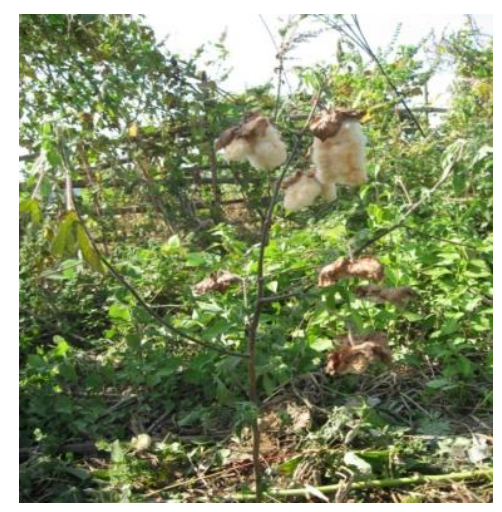


Fig.3b Capsule

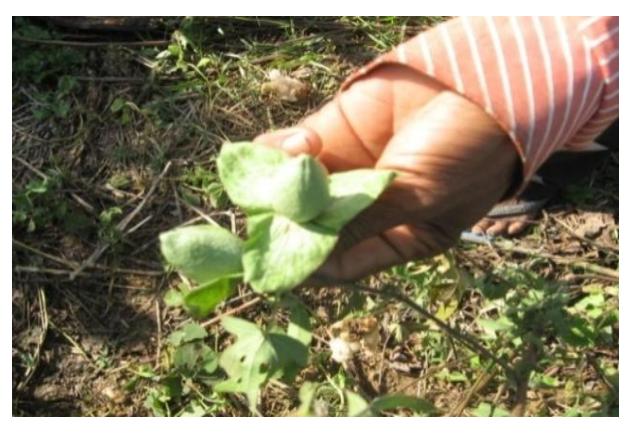

Fig.3c Brown colour lint

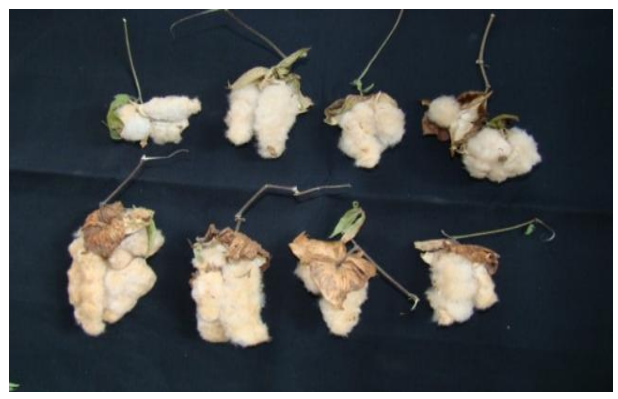

Fig.4a Desi cotton plant (Gossypium arboreum) at Uttar Barmura, Gomati district, Tripura

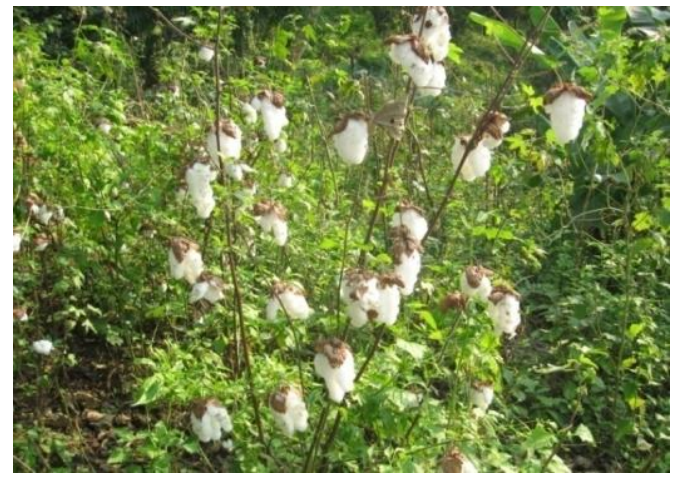

Fig.4b Capsule with burst boll and lint

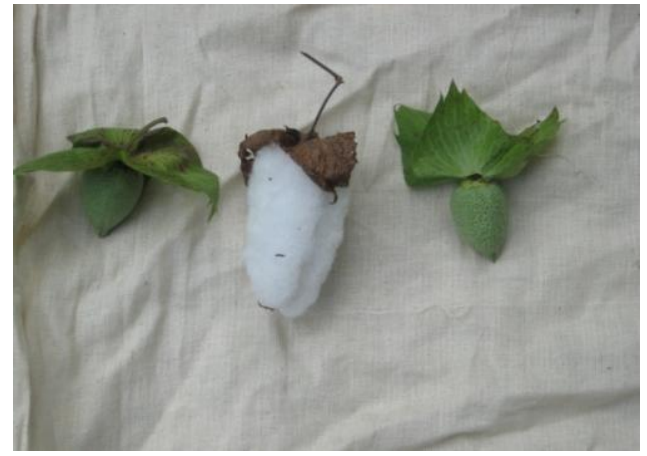


Fig.5a Gossypium barbadense in a homestead at Twirupa, Gomati district, Tripura

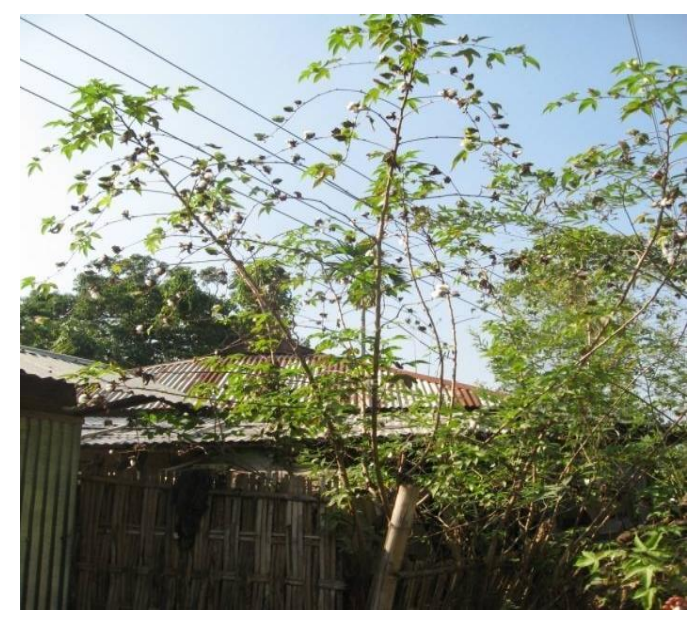

Fig.5b Leaves

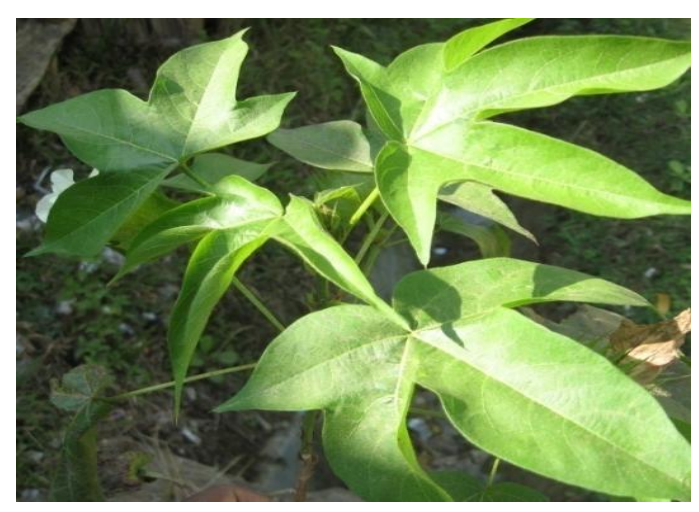

Fig.5c Twig with flower, capsule and burst boll

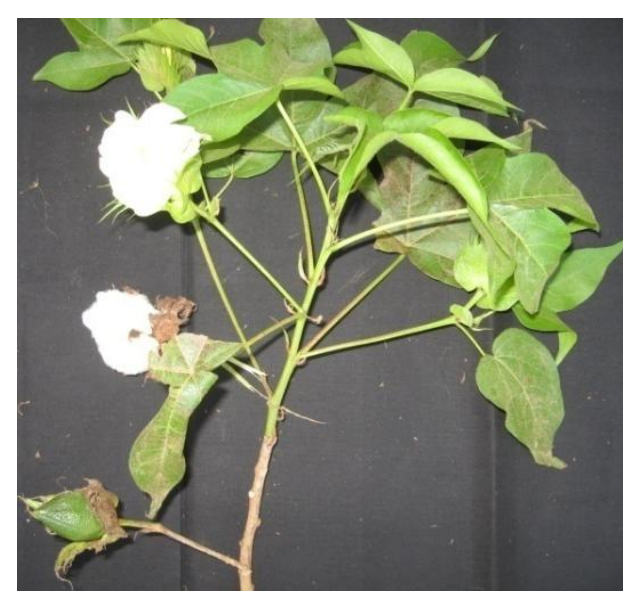


The collected cotton germplasm materials possess considerable variability in desirable traits, namely average seed cotton yield per plant (24-82 g), boll weight (4.9-7.3 g), ginning out-turn $(37-46 \%)$, mean halo length $(18-22 \mathrm{~mm})$ and resistance to various pests and diseases (Singh et al., 2003). ICARNBPGR, New Delhi collaborate with ICARCICR, Nagpur explore and collect landraces and primitive cultivars from Assam \& Manipur $(1977,1984)$ particularly $G$. arboreum race cernuum having high ginning out-turn, big \& long bolls and locule retention with high seed number (Narayanan et al., 2014). ICAR-CICR conducted exploration and collection survey to collect landraces and primitive cultivars from Arunachal Pradesh. The collected accessions represent derivatives of race 'cernuum' and race 'burmanicum' that show broad variability index for morphoeconomic characters such as leaf size, boll size and shape. The range of variability is recorded for $G$. arboreum variants of landraces with reference to fibre length (17.61-24.56mm), micronaire (5.53-7.25) and fibre strength (15.42-17.89 g/tex) (Saravanan et al., 2016). Among fourteen accessions, four are of $G$. barbadense accessions from Tripura (Fig. 5). The range of fibre quality traits for $G$. barbadense with reference to fibre length varies from 32.6 to $34.9 \mathrm{~mm}$, micronaire from 5.8 to 6.4 and fibre strength from 21.6 to 24.7 $\mathrm{g} /$ tex (Table 2). The high micronaire value in the $G$. barbadense underscored diverse germplasm material present in the collection.

The primitive cultivars and landraces of desi cotton in Tripura are under biotic influences of several kinds and magnitudes and exhibited considerable amount of shrinkage in area due to conversion of lands to rubber cultivation and rapid introduction of new varieties/Bt hybrids/ improved cultivars. These primitive cultivars are the basic source as genetically diverse material for cotton improvement and needs utmost attention for conservation. The present exploration mission was made to collect available diverse cotton accessions of desi cotton ( $G$. arboreum) and G. barbadense accessions from four districts of Tripura. The collected accessions were genetically diverse based on agro-morphological traits which may be used in cotton improvement programme. Desi coloured cotton cultivation is highly suitable for sustainable organic farming and also preparation of export oriented eco-friendly garments and cotton improvement.

\section{Acknowledgement}

Authors are grateful to the Director, ICARNBPGR, New Delhi for facilitating an exploration and germplasm collection mission to Tripura and also to the Director, ICARCICR, Nagpur for providing necessary research facilities to conduct the experiment.

\section{References}

Akhtar, K.P., Haidar, S., Khan, M.K.R., Ahmad, M. and Sarwar, N. 2010. Evaluation of Gossypium species for resistance to cotton leaf curl burewela virus. Ann. Appl. Biol. 157:135-147.

Dhawan, A.K., Simwat, G.S. and Sidhu, A.S. 1991. Field reaction of some varieties of Asiatic cotton (Gossypium arboreum L.) to sucking and boll worm pests. J.Res. Punj. Agri. Univ., Ludhiana, Punjab, India 28: 57-62.

Hutchinson, J.B., Silow, R.A. and Stephens, S.G. 1947. The evolution of Gossypium and the differentiation of the cultivated cottons. Oxford university press, London.

Kranthi, K.R. 2014. How colourful is the future of naturally coloured cotton? Cotton Statistics \& News. No.1, p.1-4.

Kulkarni, V.N., Khadi, B.M., Manjula, S.M., Deshapande, L.A. and Narayanan, S.S. 2009. The worldwide gene pools of 
Gossypium arboreum L. and $G$. Herbaceum L., and their improvement. In: Genetics and Genomics of Cotton (Paterson, A.H., ed.), pp. 69-97, Springer.

Maqbool, A., Abbas, W., Rao, A.Q., Irfan, M. and Zahur, M. 2010. Gossypium arboreum GHSP26 enhances drought tolerance in Gossypium hirsutum. Biotech. Prog. 26:21-25.

Mehetre, S.S., Aher, A.R., Gawande, V.L., Patil, V.R. and Mokate, A.S. 2003. Induced polyploidy in Gossypium: a tool to overcome interspecific compatibility of cultivated tetraploid and diploid cottons. Curr. Sci. 84:15101512.

Narayanan, S.S., Parchuri V. and Srinivasu, K,B., 2014 Cotton Germplasm in IndiaNew Trends DOI: 10.5772/58622.

Nibouche, S., Brevault, T., Klassou, C., Dessauw, D. and Hau, B. 2008. Assessment of the resistance of cotton germplasm (Gossypium spp.) to aphids (Homoptera, Aphididae) and leaf hoppers, Homoptera, Cicadellidae, Typhlocybinae): methodology and genetic variability. Plant Breed. 127:376-382.

Pareek, S.K., Bisht, I.S., Bhatt, K.C., Kumar, A., Koppar, M.N., Gupta, P.N., Mithal, S.K. and Singh. R. 2000. Manual on Exploration and Collection of plant
Genetic Resources and Related indigenous Knowledge. Jai Vigyan National Science and Technology Mission on Conservation of Agrobiodiversity (Plant Genetic Resources), ICAR-NBPGR, New Delhi, p 63.

Saravanan, M., Das, J., Misra, R.C., Mohan, P., Waghmare, V.N. and Kranthi, K.R. 2016. Genetic diversity analysis using SSR markers for desi cotton ( $G$. arboreum) landraces collected from Arunachal Pradesh. Cotton Research Journal, Vol 7, No.2:91-96.

Singh, V.V, Mohan P., Kulkarni V.N., Baitul, S.J. and Pathak, B.R. 2003. Exploration within India for collection of cotton species germplasm. Plant Genetic Resources Newsletter Issue No.136:4046.

Tahir, M.S., Khan, N.U.I and Sajid, U.R. 2011. Development of an interspecific hybrid (triploid) by crossing Gossypium hirsutum and G. arboreum. Cytologia 76:193-199.

Waghmare, V.N. and Koranne, K.D. 1998. Coloured Cotton: present status, problems and future potentials. Ind. J. Genet., 58(1):1-15.

Wheeler, T.A., Gannaway, J.R. and Keating, K. 1999. Identification of resistance to Thielaviopsis basicola in diploid cotton. Plant Dis. 83 (9):831-833.

\section{How to cite this article:}

Saravanan, M., R. C. Misra, S. S. Mahajan, P. Mohan and Waghmare, V. N. 2021. Exploration, Characterization and Conservation of Cotton (Gossypium spp.) Encompassing Colour Cotton from Tripura, India. Int.J.Curr.Microbiol.App.Sci. 10(02): 3007-3016. doi: https://doi.org/10.20546/ijcmas.2021.1002.329 